\title{
Acute Type A Dissection, Coarctation of Aorta and Levoatriocardinal Vein - A Rare Combination
}

\author{
Kartik Patel ${ }^{1}$, Archit Patel $^{1}$, and Chandrasekaran Ananthanarayanan ${ }^{2}$ \\ ${ }^{1}$ U.N.Mehta Institute of Cardiology and Research Center \\ ${ }^{2}$ U. N. Mehta Institute of Cardiology and Research Center
}

June 18, 2021

\begin{abstract}
Concomitant presence of acute type A dissection and coactation of aorta is rare (1). Levoatriocardinal vein has shown to be associated with left sided hypoplastic lesions as well as with normal hearts $(2,3)$. However, concurrent presence of levoatriocardinal vein with acute type A dissection, severe aortic regurgitation and Coarctation of aortic isthmus was not described. We here described a case of 20 year male presented to emergency department with acute chest pain radiating to back. On evaluation, he was found to have acute type A dissection with dilated aortic root, severe aortic regurgitation, normal mitral valve, severe coarctation of aorta and levoatriocardinal vein. Patient was managed successfully with composite valve conduit replacement of ascending aorta with ascending aortic to descending aortic graft (16mm graft) with levoatriocardinal vein ligation.
\end{abstract}

\section{Acute Type A Dissection, Coarctation of Aorta and Levoatriocardinal Vein - A Rare Combi- nation}

Kartik Patel M.Ch ${ }^{1}$, Archit patel, M.Ch ${ }^{2}$, C Anathnarayan M.Ch ${ }^{3}$

U. N. Mehta Institute of Cardiology and Research Center (affiliated to BJ medical college, Ahmedabad)

Departmental affiliation of authors:

1. Associate Professor, Department of Cardiovascular and Thoracic Surgery

2. Assistant Professor, Department of Cardiovascular and Thoracic Surgery

3. Ex.Assistant Professor, Department of Cardiovascular and Thoracic Surgery

Address for Correspondence:

Dr. Kartik Patel, Associate Professor

Department of Cardio Vascular and Thoracic Surgery,

Email: drkartikpgi@gmail.com

M: 91-9925249221; Fax: 079-22682092

U. N. Mehta Institute of Cardiology and Research Centre,Civil Hospital Campus, Asarwa,

Ahmedabad-380016, Gujarat, India

Word count: 450

Funding

The study did not receive any funding. 


\section{Conflict of interest statement}

All authors declare that they have no conflict of interest and do not receive any research grants from any company, have not received a speaker honorarium from any company, do not own any stock in any company and are not members of a committee.

Authors' Statement - The patient consented to the submission and publication of this report.

IRB Number : As per our Institute protocol we don't have to approve the (Image and Case report) study before publication. We had taken the consent from the patient prior to submission.

\section{Main text}

Concomitant presence of acute type A dissection and coactation of aorta is rare (1). Levoatriocardinal vein has shown to be associated with left sided hypoplastic lesions as well as with normal hearts $(2,3)$. However, concurrent presence of levoatriocardinal vein with acute type A dissection, severe aortic regurgitation and Coarctation of aortic isthmus was not described. After taking consent from the patient we here described a case of 20 year male presented to emergency department with acute chest pain radiating to back. On evaluation, he was found to have acute type A dissection with dilated aortic root, severe aortic regurgitation, normal mitral valve, severe coarctation of aorta and levoatriocardinal vein. Patient was managed successfully with composite valve conduit replacement of ascending aorta with ascending aortic to descending aortic graft (16mm graft) with levoatriocardinal vein ligation.

\section{Figure Legends:}

Figure 1: A: Contrast enhanced computed tomography showing ascending aortic aneurysm with type A dissection along with severe coarctation of aortic ishmus. B: Contrast enhanced computed tomography showing levoatriocardinal vein communicating left atrium to left innominate vein

Figure 2: A: Post-operative contrast enhanced computed tomography showing composite valve conduit with patent ascending to descending graft. B: Intra -operative image showing complete repair

\section{References:}

1. Svensson LG. Management of acute aortic dissection associated with coarctation by a single operation. The Annals of thoracic surgery 1994 Jul 1;58(1):241-3.

2. Amoretti F, Cerillo AG, Chiappino D. The levoatriocardinal vein. Pediatric cardiology 2005 Aug; 26 (4):494-5.

3. Patel K, Kakkar D, Ananthnarayan C, Patel R, Patel D, et al. Levoatriocardinal Vein With Multiple Ventricular Septal Defects and Without Left-Sided Valvular Atresia. World Journal for Pediatric and Congenital Heart Surgery 2021 Jan; 12(1):128-30. 

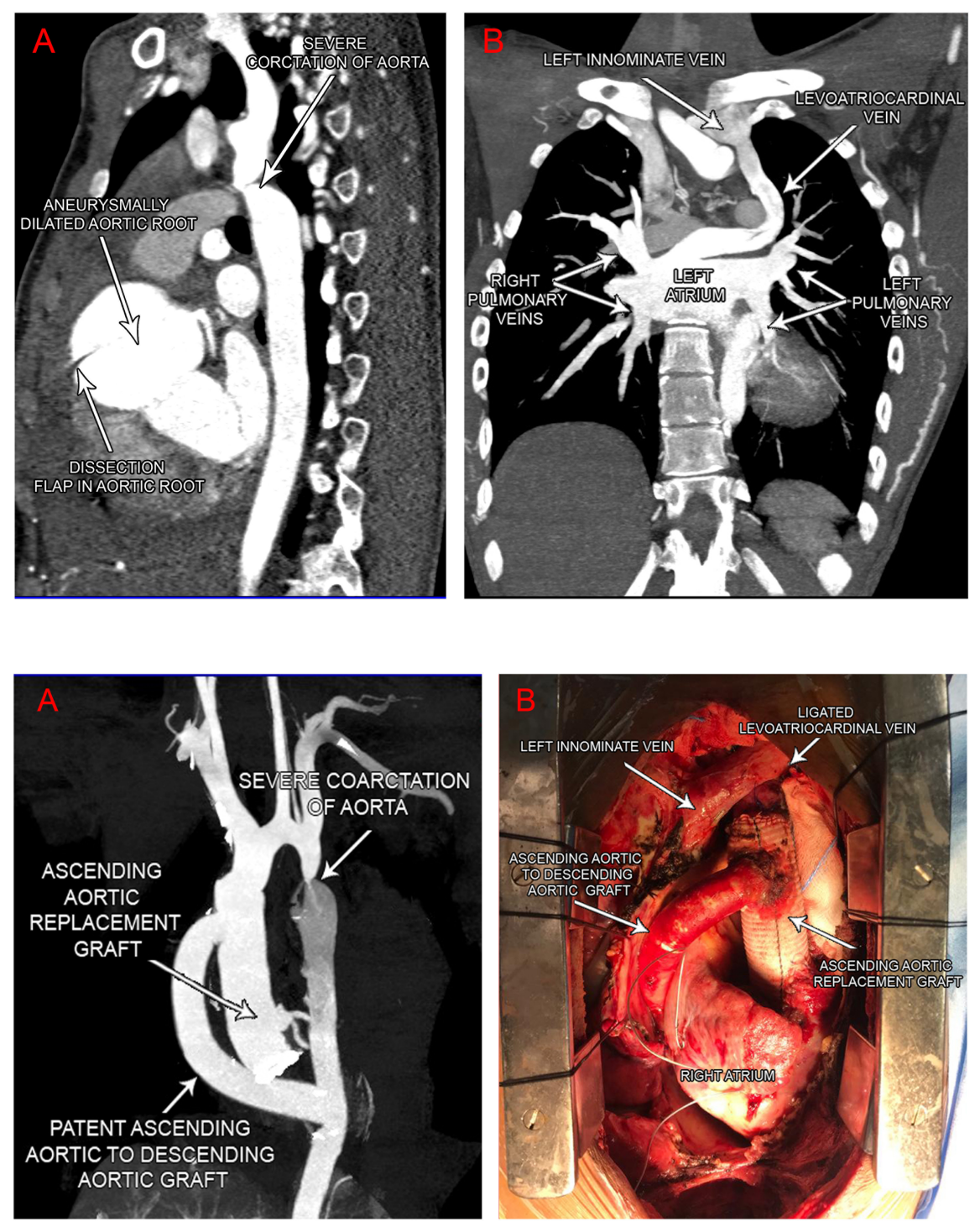